\title{
Sonographic evaluation of subacromial space
}

\author{
R. Azzoni *, P. Cabitza, M. Parrini \\ Department of Orthopaedic, State University of Milan, Department of Medical and Surgical Science, 30 Morandi Street, \\ 20097 San Donato milanese (MI), Italy
}

\begin{abstract}
Goal of the study. The purpose of this study is to compare the accuracy of sonographic to radiographic measurements of subacromial space, and verify its variations in relation to acromial morphology, age, sex and rotator cuff pathologies. Materials and methods. As a result, we have compared a radiographic examination to sonographic examination, each measuring the subacromial space in 200 random shoulders, with a personal method. The sonographic examination was performed by using a HDI 5000 ultrasound scanner Sono-CT with 7.5 MHz linear array transducer. No stand-off pad was utilized. Results. The statistical analysis of the data derived from the two measurements was not sufficient to conclude that the two techniques are different ( $p>0.8)$. They also correspond with the radiographic morphology of the acromion. The size of subacromial space was related to the acromial morphology, female gender, and rotator cuff pathology, however, it was not related to age. Discussion and conclusions. Our results clearly show that sonographic measurements are very close to those obtained by X-ray $(p>0.8)$. The Bland-Altman analysis showed that for all groups, the were small enough to give us confidence that the sonographic technique may be used in place of the radiographic one for clinical purposes. One-way ANOVA showed that sonographic measurements were statistically different among the four groups $(p<0.05)$. The sonography demonstrated precision, accuracy and carefulness in the measurement of the subacromial space.
\end{abstract}

(C) 2003 Elsevier B.V. All rights reserved.

Keywords: Sonography; Shoulder; Subacromial space

\section{Introduction}

Most of the patients with shoulder pain show a particular morphology of the anterior prominence of the acromion that reduces the subacromial space. In 1993, Wuh and Snyder [1] classified three types of acromion on the basis of a morphological study of shoulders of cadavers. Type A of acromion with an inferior protrusion of $8 \mathrm{~mm}$, type B with an inferior protrusion of $8-12$ $\mathrm{mm}$, and type $\mathrm{C}$ with an inferior protrusion of more than $12 \mathrm{~mm}$. Wuh and Snyder as well as other authors $[2,3]$ found that a greater percentage of subjects with a type $\mathrm{C}$ acromion are affected by rotator cuff tears [4].

Radiographic evaluation of shoulder morphology is therefore useful for most diagnosis; the antero-posterior view and the outlet view allow a good evaluation of bone configuration and their relationships.

${ }^{*}$ Corresponding author. Fax +39-0252-774632.

E-mail address: roberto.azzoni@unimi.it (R. Azzoni).
In 1994, Flatow et al. [5] assessed the subacromial space of a normal X-ray, quantifying it as being an average of $10-15 \mathrm{~mm}$.

Given the importance of the morphology of the acromion and the size of the subacromial space in the onset of shoulder pain, with this study, our purpose is to also confirm the accuracy of sonographic to radiographic measurements of subacromial space. The accuracy of these two techniques has been considered in relation to age, sex and rotator cuff pathologies.

\section{Materials and methods}

Between 1 March 2001 and 28 February 2002 (1 year) as part of a prospective study, we examined with 200 random shoulders pain (200 patients who experienced), using the sonographic technique described by Peetrons [6], and measured the subacromial space in all the cases by using our own method, subsequently described. The echography and X-ray exams were performed always on both shoulders for comparison. 
Sonography was performed using a HDI 5000 ultrasound scanner Sono-CT (ATL-Philips, Bothell, WA) with $7.5 \mathrm{MHz}$ linear array transducer. Sono-CT is a software method of real time spatial compounding, averaged over nine frames which are acquired at different angles across the transducer aperture. No stand-off pad was utilized. The images were recorded using UP895 Video printer UP-895 CE (SONY, Tokyo, Japan). This is a standard method of recording, usually employed in clinical practice, and the images are used for diagnoses of rotator cuff pathologies.

The measurement of subacromial space were made using coronal axis views of the shoulder. The patient was sat with his upper limb in backward flexion and internal rotation; the upper limb was rested on the surface of the bed to prevent muscle contraction, by reason of humeral head climb [7]. The acromion itself and the head of the humerus were taken at greater tuberosity level as the points of reference, which allowed optimal visualisation of the long axis view of the supraspinatus.

The sonographic callipers for the measurements were placed on the most inferior echo from the external and inferior margin of the acromion and on the nearest point of the echo of the surface of the humeral head, that yielded the smallest distance. Three different pictures were taken of all of the shoulders and, for this study, we always used the picture showing the greatest distance. Measurements were performed by the authors during sonographic exam. Flatow et al. [5] assessed the normal $\mathrm{X}$-ray subacromial space, quantifying it as being an average of $10-15 \mathrm{~mm}$. Other authors measure subacromial space with an MRI [7] (values $8.2 \mathrm{~mm}$ in men and 7 $\mathrm{mm}$ in women at $30^{\circ}$ degrees of abduction) and with intra-operative measurement obtained by artroscopy [8] $(16 \mathrm{~mm})$ : the values of the subacromial distance measured were very different in relation to the method of evaluation.

Our radiographic measurements were obtained according to Flatow's [5] and Umans's [9] methods.

All patients studied had a history of pain. Some of them also experienced weakness and stiffness of the shoulder for a period of months. Some of the patients had a history of trauma. Fifteen patients were involved in sports: golf ( 7 cases), tennis (6 cases), volley-ball ( 2 cases).

The 200 shoulders were divided into four groups on the basis of rotator cuff conditions: sonographic normal shoulders (group 1), shoulders with tendinopathy but without cuff tears (group 2), shoulders with partial tears limited to the supraspinatus (group 3), and shoulders with complete tears of the rotator cuff (supraspinatus and subscapular) (group 4).

A normal rotator cuff was diagnosed when its image demonstrated tendons echogenics, homogeneous, curves with normal thickness laying on the opposite side and with absence of internal hypo-echoic or anechoic areas (Fig. 1).

The tendinopathy of rotator cuff is considered an image of tendons with abnormal thickness (increased or reduced as to oedema and inflammation or to involution), and with absence of internal hypo-echoic or anechoic areas (Fig. 2).

The partial tears of a rotator cuff (limited to the supraspinatus) is considered an image with presence of

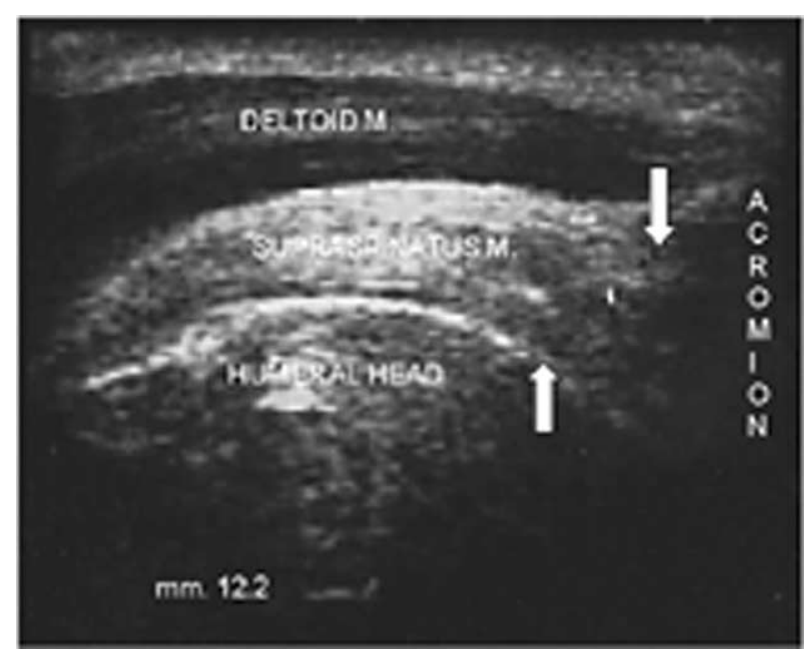

Fig. 1. US picture in long axis view (coronal view) of normal supraspinatus tendon: tendon is echogenic, homogeneous, curve, with normal thickness, laying on the opposite side and with absence of internal hypo-echoic or anechoic areas. Shown callipers (arrows) for the measurements placed on the most inferior echo from the external margin of the acromion and on the nearest point of the surface of the humeral head: distance is $12.2 \mathrm{~mm}$.

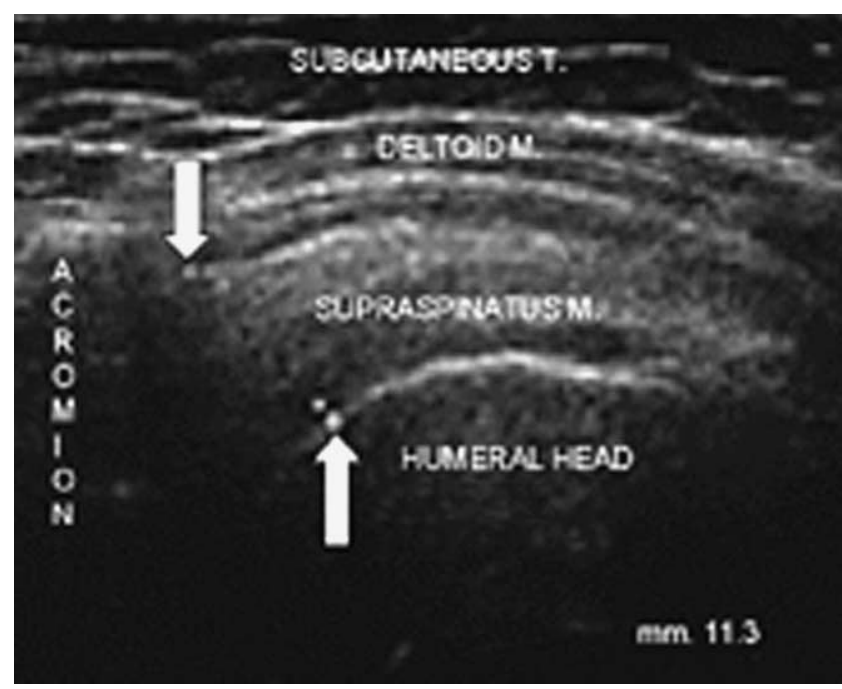

Fig. 2. US picture in long axis view (coronal view) of shoulder with tendinopathy of supraspinatus: tendon looks of abnormal thickness (increased due to oedema and inflammation), hypo-echoic, and absence of internal hypo-echoic or anechoic areas. Shown callipers (arrows) for the measurements and references like Fig. 1: distance is 11.3 $\mathrm{mm}$. 
hypo-echoic or anechoic areas of tendon with a nonhomogeneous echo-structure (Fig. 3).

The cuff with complete tears (supraspinatus and subscapular) demonstrates a very significant reduction of thickness of the tendons so much that they almost disappear (Fig. 4).

Before or after sonography, all of the subjects underwent a X-ray examination of the shoulders using antero-posterior, trans-axillary and supraspinatus outlet views in order to study the morphology of the acromion

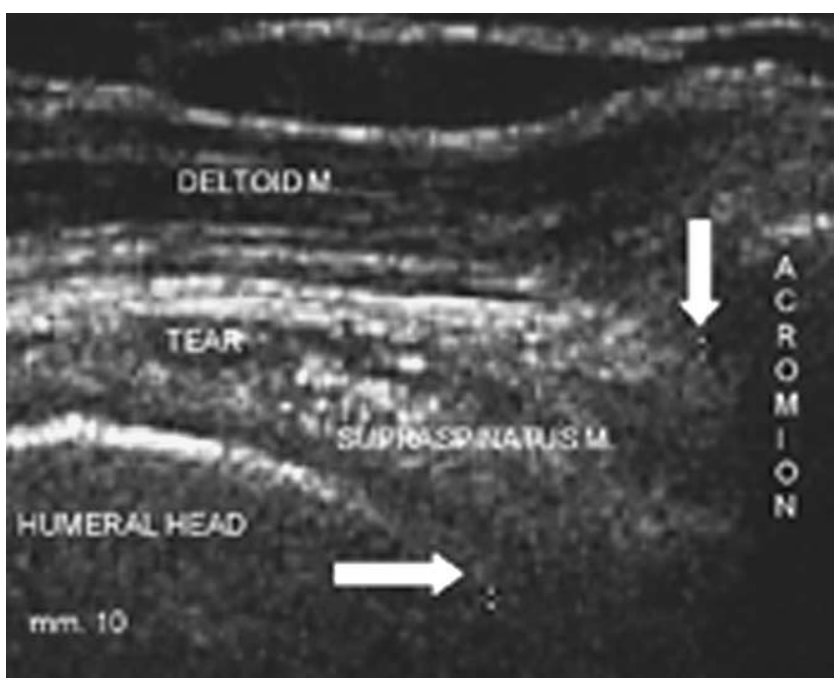

Fig. 3. US picture in long axis view (coronal view) of shoulder with partial tears of supraspinatus: inside tendon look a presence of hypoechoic area with a non-homogeneous echo-structure. Shown callipers (arrows) and references for the measurements like Fig. 1: distance is $10.0 \mathrm{~mm}$.

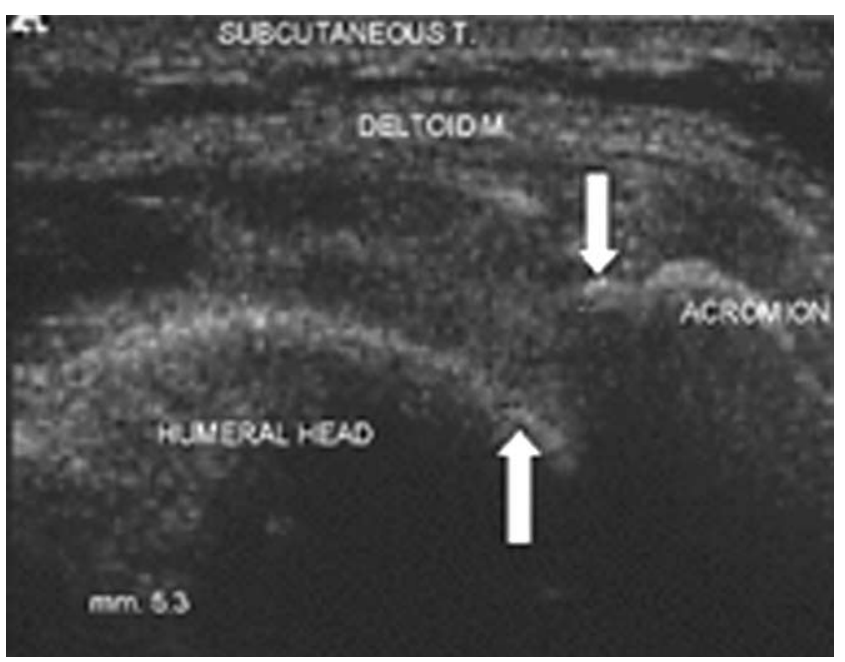

Fig. 4. US picture in long axis view (coronal view) of shoulder with a complete tear of supraspinatus tendon: looks like a very important reduction of thickness of tendon nearly disappeared. Shown callipers (arrows) and references for the measurements like Fig. 1: distance is 5.3 $\mathrm{mm}$. and any osteoarthritic alterations of the acromioclavicular joint, as well as measure the subacromial space [5].

\section{Statistical analysis}

The gathered data has been reported in the form of a mean and a range. Relationships among parameters have been estimated by using the Pearson's correlation coefficient $r$ and a regression analysis.

The $t$-test and, when necessary, a one-way analysis of variance (ANOVA) have been used to compare data.

Furthermore, in order to compare the two techniques, a Bland-Altman analysis [10] has been used. Briefly, in this method, the differences between the two measurements are plotted against the averages of the two measurements. If the differences within \pm 1.96 standard deviation from the mean are not clinically important, the two methods may be used inter-changeably.

Statistical analysis was done in Excel and MedCalc software for Windows XP.

In all tests, we have assumed that 0.05 indicated a statistically significant difference.

\section{Results}

Our echographic method of measurement of subacromial space is reproducible, as 24 measures of 25 healthy subjects, recorded by two different operators, showed a correlation coefficient $r=0.8, p<0.05$.

Group 1 consisted of 38 females and 32 males between the ages of 20 and 62 years old (mean age 48.6 years) whose painful shoulder (right in 38 cases and left in 32; dominant in 40 cases) showed a normal sonographic picture of the rotator cuff, and a subacromial space width of between 6.9 and $16.6 \mathrm{~mm}$ (Fig. 1). The $\mathrm{X}$-ray measured subacromial space was $11.0 \mathrm{~mm}$.

Group 2 consisted of 22 females and 32 males between the ages of 33 and 80 years old (mean age 57.5 years) whose painful shoulder (right in 34 cases and left in 20; dominant in 40 cases) showed an sonographic picture of rotator cuff tendinopathy without any sure signs of tears, and a subacromial space width of between 9.6 and $17.4 \mathrm{~mm}$ (Fig. 2). The X-ray measured subacromial space was $13.9 \mathrm{~mm}$.

Group 3 consisted of 14 females and 6 males between the ages of 53 and 76 years old (mean age 64 years) whose painful shoulder (right in 10 cases and left in 10; dominant in 15 cases) showed a sonographic image of a partial supraspinatus tendon tear and a subacromial space width of between 6.5 and $13.1 \mathrm{~mm}$ (Fig. 3). The $\mathrm{X}$-ray measured subacromial space was $10.1 \mathrm{~mm}$.

Finally, group 4 consisted of 10 females and 16 males between the ages of 45 and 73 years old (mean age 60.4 
years) whose painful shoulder (right in 16 cases and left in 10; dominant in 18 cases) showed an sonographic image of a complete (supraspinatus and subscapular) tear of the rotator cuff, and a subacromial space width of between 6.1 and $12.9 \mathrm{~mm}$ (Fig. 4). The X-ray measured subacromial space was $9.0 \mathrm{~mm}$.

The radiographic pictures in group 1 indicated type B acromonial morphological abnormalities in 24 shoulders (with sonographic and radiographic subacromial spaces of, respectively, 9.4 and $10.0 \mathrm{~mm}$ ) and type $\mathrm{C}$ abnormalities in 12 shoulders (sonographic and radiographic subacromial spaces of, respectively, 8.5 and $7.7 \mathrm{~mm}$ ); none of the radiographic images suggested the presence of acromioclavicular osteoarthritis.

Group 2 included 18 cases with a radiographic type B acromions (with sonographic and radiographic subacromial spaces of, respectively, 10.5 and $11.0 \mathrm{~mm}$ ) and 14 cases with type $\mathrm{C}$ acromions (sonographic and radiographic subacromial spaces of, respectively, 9.4 and $9.3 \mathrm{~mm}$ ); there were radiographic indications of acromioclavicular osteoarthritis in three cases.

Group 3 included two radiographic type B acromions (with sonographic and radiographic subacromial spaces of, respectively, 10.0 and $10.3 \mathrm{~mm}$ ) and six type $\mathrm{C}$ acromions (sonographic and radiographic subacromial spaces of, respectively, 7.2 and $6.5 \mathrm{~mm}$ ); there were radiographic indications of acromioclavicular osteoarthritis in one case.

Finally, group 4 included four radiographic type B acromions (with sonographic and radiographic subacromial spaces of, respectively, 10.5 and $9.8 \mathrm{~mm}$ ) and 20 type $\mathrm{C}$ acromions (sonographic and radiographic subacromial spaces of, respectively, 7.8 and $7.0 \mathrm{~mm}$ ); there were radiographic indications of acromioclavicular osteoarthritis in one case.

There was a significant correlation between the sonographic and radiographic measurements of the subacromial space (group 1: $r=0.85$, group 2: $r=0.82$, $p<0.01$; group 3: $r=0.80$, group 4: $r=0.77, p<0.5$ ), as well as between these and the X-ray type acromion itself ( $r>0.75, p<0.05$ for each group). Furthermore, each paired $t$-test showed $p>0.08$.

The Bland-Altman analysis showed that there was a mean difference of -0.3 and limits of agreements: -2.2 and 1.5 for group 1; 0.8 and limits: -1.6 and 3.1 for group $2 ;-2.4$ and limits: -1.5 and -3.3 for group $3 ;-2.4$ and limits: -1.4 and -3.4 for group 4 . Since, the limits of the agreements for each group were small enough, we can be confident that the sonographic technique may be used in place of the radiographic for clinical purposes.

One-way ANOVA showed that sonographic measurements were statistically different among the four groups $(p<0.05)$. Furthermore, the mean values of female patients were significantly reduced $(p<0.05)$ with respect to male, for each group.
On the contrary, no correlation was found between sonographic measurements and the age of patients, for each group.

\section{Discussion}

The measurement of subacromial space width is important in the study of normal and pathologic shoulders.

Our results clearly show that sonographic measurements are very close to those obtained by X-ray $(p>0.8)$. The Bland-Altman analysis showed that the limits of the agreements for each group were small enough to give us confidence that the sonographic technique may be used in place of the radiographic one for clinical purposes. One-way ANOVA showed that sonographic measurements were statistically different among the four groups $(p<0.05)$. Furthermore, the mean values of female patients were significantly reduced $(p<0.05)$ with respect to male patients, for each group.

The subacromial space width is smaller in nearly all rotator cuff pathologies, but becomes even smaller as the severity of the condition increases and is smaller in the case of a complete cuff tear. As could be logically expected, the reduction in the space width is related to the climb of the humeral head because of the action of the deltoid muscle. Other significant findings are that the average distance of the space width is smaller in females $(p<0.05)$ and that the prevalence of cuff's tendons pathologies is greater on the dominant side [11].

The limitation of the method is that it does not measure a potentially smaller of subacromial space width due to the presence of acromio-clavicular osteoarthritis because it does not allow the measurement of the inferior protrusion of the acromio-clavicular joint where the penetration of beams is impossible.

In conclusion, in the light of our results, as sonography has proved to be an extremely sensitive screening method for differential diagnosis of ruptures of the rotator cuff [12], we think it is useful to complete the sonographic examination of the shoulder by measuring carefully the subacromial space. Beginning the diagnosis of a painful shoulder via sonography, the subacromial space measurement can avoid radiographic examination of the shoulder, which would result in reduction of cost as well as patient exposure to radiation. The advantage of the sonography is that it has a higher resolution, it provides the possibility of a dynamic examination and an easier comparison with the opposite side. Moreover, since sonography is inexpensive, non-invasive and already widely used for the diagnosis of rotator cuff pathologies, we thought it might be useful to evaluate the reliability of our technically simple and rapid method of using it to measure subacromial space during 
the course of a routine sonographic examination of the shoulder. Finally statistical analysis show that the sonographic technique may be used in place of the radiographic technique for clinical purposes.

\section{References}

[1] H.C.K. Wuh, S.J. Snyder, A modified classification of the supraspinatus outlet view based on the configuration and the anatomic thickness of the acromion, Orthop. Trans. 16 (19921993) 767-772.

[2] D.A. Toivonen, M.J. Tuite, J.F. Orwion, Acromial structure and tears of the rotator cuff, J. Shoulder Elbow Surg. 4 (1995) 376383.

[3] L.U. Bigliani, D.S. Morrison, E.W. April, The morphology of the acromion and its relationship to rotator cuff tears, Orthop. Trans. 10 (1986) 228-231.

[4] D.S. Morrison, L.U. Bigliani, The clinical significance of variations in acromial morphology, Orthop. Trans. 11 (1987) 234-240.
[5] E.L. Flatow, L.J. Soslowsky, J.B. Ticker, et al., Excursion of the rotator cuff under the acromion. Patterns of subacromial contact, Am. J. Sports Med. 22 (1994) 779-788.

[6] P. Peetrons, Epaule, in: B.D. Fornage, Echographie des membres, Paris, F. Vigot, 1991, pp. 121-128 (French).

[7] H. Graichen, H. Bonel, T. Stammberg, et al., Sex-specific differences of subacromial space width during abduction, with and without muscular activity, and correlation with anthropometric variables, J. Shoulder Elbow Surg. 10 (2001) 129-135.

[8] B. Tillander, R. Norlin, Intraoperative measurements of subacromial distance, Arthroscopy 18 (2002) 347-352.

[9] H.R. Umans, H. Pavlov, M. Berkowitz, R.F. Warren, Correlation of radiographic and arthroscopic findings with rotator cuff tears and degenerative joint disease, J. Shoulder Elbow Surg. 10 (2001) 428-433.

[10] J.M. Bland, D.G. Altman, Statistical method for assessing agreement between two methods of clinical measurement, Lancet 8 (1986) 307-310.

[11] C. Wurning, Shoulder impingement (Germany), Orthopade 29 (2000) 868-880.

[12] B. Dubs, Ultrasound study of the shoulder (Germany), Schweiz. Runds. Med. Praxis 90 (2001) 667-671. 